Research Article

\title{
Rolling Bearing Fault Vibration Signal Denoising Based on Adaptive Morphological Wavelet Perona-Malik Filter Algorithm
}

\author{
Hao Li $\mathbb{D}^{1,2}$ Yifan Tan $\left(\mathbb{D},{ }^{1}\right.$ and Yun Pu $\mathbb{D}^{1}$ \\ ${ }^{1}$ Southwest Jiaotong University School of Transportation and Logistics, Chengdu 610031, China \\ ${ }^{2}$ Officers College of PAP, Chengdu 610213, China \\ Correspondence should be addressed to Yifan Tan; yftan@my.swjtu.edu.cn and Yun Pu; ypu@swjtu.edu.cn
}

Received 22 January 2021; Revised 29 June 2021; Accepted 19 November 2021; Published 13 December 2021

Academic Editor: Gang Tang

Copyright (C) 2021 Hao Li et al. This is an open access article distributed under the Creative Commons Attribution License, which permits unrestricted use, distribution, and reproduction in any medium, provided the original work is properly cited.

\begin{abstract}
This paper proposes an adaptive Perona-Malik filtering algorithm based on the morphological Haar wavelet, which is used for vibration signal denoising in rolling bearing fault diagnosis with strong noise. First, the morphological Haar wavelet operator is utilized to presmooth the noisy signal, and the gradient of the presmooth signal is estimated. Second, considering the uncertainty of gradient at the strong noise point, a strong noise point recognition operator is constructed to adaptively identify the strong noise point. Third, the two-step gradient average value of the strong noise point in the same direction is used to substitute, and the second derivative is introduced into the diffusion coefficient. Finally, diffusion filtering is performed based on the improved Perona-Malik model. The simulation experiment result indicated that not only the algorithm can denoise effectively, but also the average gradient and second derivative in the same direction can effectively suppress the back diffusion of strong noise points to improve the denoising signal-to-noise ratio. The experimental results of rolling bearing show that the algorithm can adaptively filter out strong noise points and keep the information of peak in the signal well, which can improve the accuracy of rolling bearing fault diagnosis.
\end{abstract}

\section{Introduction}

In the mechanical fault diagnosis based on the vibration signal, in order to extract fault features more accurately, it is a crucial step to denoise the vibration signal and reduce noise interference. Since the running state of mechanical equipment is time-varying, speed and load are unstable due to the working demand, especially when a fault occurs. The stiffness, damping, and elastic force of the system would change; hence, the vibration signal shows the characteristics of nonlinear and nonstationary. In addition, measured signal is disturbed by all kinds of noise on the scene, which makes it more difficult to achieve the ideal effect by using the traditional signal processing method.

Currently, the denoising methods for mechanical vibration signals are varied [1], including time domain, frequency domain, time-frequency domain, linear, and nonlinear. These methods are different in principle and have their own characteristics. STFT is based on the assumption of piecewise signal stationarity, which is suitable for the analysis of varying signals slowly; however, it is difficult to achieve good results for the drastic varying signals. Wavelet transform plays a very important role in mechanical fault diagnosis, but the selection of wavelet basis is a difficult issue. The morphological filtering method is closely to the selection of structural elements; once the structural elements are not selected properly, it would be counterproductive and cannot play a good denoising effect and even lose the details of the signal. Generally, these methods all have a common problem, that is, some useful feature information may be removed while denoising; furthermore, signal denoising and feature extraction cannot be achieved simultaneously [2-5]. How to denoise effectively and retain feature information has always been a hot topic.

Partial differential equation (PDE) denoising theory and method is an image/signal processing method proposed in recent years, which are extensively utilized in image denoising, image enhancement, and other image processing fields. The biggest feature of the PDE method is that both image denoising and edge feature information preservation 
are considered [6-11]. Among the PDE denoising methods [6], the Perona-Malik model, the focus of present scientific research, is one of the most classic nonlinear anisotropic diffusion filtering models, in which the principle and calculation are relatively simple. At present, some scholars have created a lot of algorithms based on the Perona-Malik model [12-19]. And the application of these algorithms in image processing and other fields has achieved incomparable effects with classical algorithms. But there are few relevant studies about the research and application of the PDE denoising method in the field of mechanical vibration signal processing. The Perona-Malik model was applied in Grinding Machine and Numerical Control Machine for vibration signal denoising by $\mathrm{Wu}$ et al. and had great results. The superiority of PDE was magnified when compared with the traditional denoising method [20,21]. Moreover, PDE can be used for purification of the shaft centerline orbit [22]. $\mathrm{Xu}$ et al. [23] proposed an adaptive denoising method for vehicle's acceleration signal based on PDE. Lan et al. [24] provided a new denoising method to suppress white noise interference in GIS PD signals based on PDE. It showed the effectiveness of PDE. Among them, the proposed model [18] discussed the vibration signal denoising approach based on the Perona-Malik model and investigated the feasibility and effectiveness of the proposed method. However, due to the reverse diffusion issue of strong noise points, it has affected its wide use to some extent and needs to be further improved.

In this paper, the Perona-Malik model is improved and introduced into rolling bearing fault vibration signal denoising, which makes a contribution in the field of mechanical rolling bearing fault diagnosis. First, the morphological Haar wavelet is used to presmooth the noisy vibration signal, and the strong noise recognition operator is designed to identify the strong noise points. Second, the same direction average gradient is used to adaptively remove the strong noise points, and the second derivative is further introduced into the diffusion coefficient. Finally, the improved Perona-Malik model is utilized to complement the diffusion filtering and denoising on simulated signals and measured rolling bearing vibration signals.

The main contributions of this paper are as follows:

(1) The Perona-Malik filter model of the morphological Haar wavelet is proposed, and the second derivative is introduced into the diffusion coefficient function of the morphological Haar wavelet.

(2) A strong noise recognition operator is constructed for adaptive recognition of strong noise points, and a codirectional mean gradient is introduced for adaptive removal of strong noise points

(3) Based on the denoising results of rolling bearing fault simulation signals, the wavelet threshold denoising method, morphological Haar wavelet method, Perona-Malik model, Catté model, and the denoising performance of the improved algorithm are compared, and the effectiveness of the improved algorithm is demonstrated

(4) Denoising and spectrum analysis were performed on the fault vibration data of drive motor bearing inner ring from the Electrical Engineering Laboratory of Case Western Reserve University to verify the feasibility of applying the improved algorithm to engineering practice

\section{Perona-Malik Model}

PDE denoising processes the signals based on the motion point of view, which originates from the initial value problem of the heat conduction equation. Perona and Malik first proposed a nonlinear anisotropic diffusion model, i.e., the Perona-Malik model [6].

$$
\left\{\begin{array}{l}
\frac{\partial u(x, t)}{\partial t}=\frac{\partial}{\partial x}\left(g\left(\left|\frac{\partial u}{\partial x}\right|\right) \frac{\partial u}{\partial x}\right), \quad \forall(x, t) \in R \times(0, \infty) \\
u(x, 0)=u_{0}(x), \quad x \in R
\end{array}\right.
$$

where $g(\cdot)$ indicates the diffusion coefficient and is a monotone nonincreasing function with the conditions of $g(0)=1$ and $\lim _{s} g(s)=0$. The diffusion coefficient $g(|\partial u / \partial x|)$ is a nonnegative function of the gradient mode $|\partial u / \partial x|$, which is the core of the Perona-Malik model to control the amount and direction of diffusion.

The classic diffusion coefficient is presented as follows:

$$
\begin{aligned}
& g(s)=\exp \left(-\frac{s^{2}}{k^{2}}\right), \\
& g(s)=\frac{1}{\left(1+\left(s^{2} / k^{2}\right)\right)},
\end{aligned}
$$

where $k$ is the gradient threshold parameter. If the threshold $k$ is given, then the area of $|\partial u / \partial x|<k$ is considered to be the flat area of the signal, and the diffusion is positive and faster, which is beneficial to denoise; the area of $|\partial u / \partial x| \geq k$ is considered to be the detail feature area of the signal, and the diffusion is reverse and slower; thus, the signal is sharpened, which is conducive to the preservation of signal characteristics and can even be enhanced. These results bring great limitations to the application of the Perona-Malik model in filtering: the noise level is relatively large, especially when the signal is interfered by impulsive noise, a large gradient often exists at the noise point, and then the noise cannot be removed but may be enhanced, which is contrary to the idea of filtering. In order to avoid these characteristics of the Perona-Malik model, it is necessary to construct a new diffusion coefficient function and improve the Perona-Malik filter model. 


\section{Morphological Wavelet Perona-Malik Filter Model}

3.1. Diffusion Coefficient Function of the Catté Operator. Catté et al. modified the diffusion coefficient function of the Perona-Malik model and proposed the Catté operator $[25,26]$ as

$$
g(s)=\exp \frac{\left\{-\partial\left(G_{\sigma} * s\right) /\left.\partial x\right|^{2}\right\}}{K^{2}},
$$

where $G_{\sigma}$ is the Gaussian weighted low-pass filter with a standard deviation of $\sigma$. Then, the satisfiability of the solution was proved. The main idea of the Catte operator can be summarized as follows: considering that the signal itself contains noise, the signal is pre-Gaussian-smoothed before calculating the diffusion amplitude, which allows the diffusion process to remove small features in the high-contrast area. Essentially, the Catté operator is a linear low-pass Gaussian filter, in which the signal may be excessively smoothed. In order to solve this limitation, a nonlinear filter operator must be used to replace the linear low-pass Gaussian filter.

3.2. Diffusion Coefficient Function of Morphological Haar Wavelet. This paper utilizes the morphological Haar wavelet operator (nonlinear filter operator) to replace the linear Gaussian filter of the Catté operator. The morphological Haar wavelet diffusion coefficient function is

$$
g(s)=\exp \frac{\left\{|\partial(M s) / \partial x|^{2}\right\}}{K^{2}}
$$

where $M s$ represents the morphology Haar wavelet filtering of the signal $s$.

Morphological Haar wavelet is a nonlinear wavelet transform based on mathematical morphology. It is a nonlinear extension of the morphological operator to the linear Haar wavelet [27], which can retain edge information more effectively than linear wavelets. Meanwhile, the morphological Haar wavelet has the nonlinear analysis characteristics of mathematical morphology and the multiresolution characteristics of wavelets $[28,29]$.

The analysis and composition operator of the morphological Haar wavelet is presented as follows:

$$
\left\{\begin{array}{l}
\psi^{\uparrow}(x)(n)=x(2 n) \wedge x(2 n+1), \\
\omega^{\uparrow}(x)(n)=x(2 n)-x(2 n+1), \\
\psi^{\downarrow}(x)(2 n)=\psi^{\downarrow}(x)(2 n+1)=x(n), \\
\omega^{\downarrow}(y)(2 n)=y(n) \vee 0, \omega^{\downarrow}(y)(2 n+1)=-[y(n) \wedge 0] .
\end{array}\right.
$$

The operation of the morphological Haar wavelet analysis operator is minimal, and the operation process of the morphological Haar wavelet only includes extreme value and addition and subtraction operations, which also simplifies the calculation and improves the processing speed.
In order to better preserve the meaningful and strong spike and boundary details in the signal, the second derivative can be further introduced into the morphological Haar wavelet diffusion coefficient as

$$
g(s)=\exp \frac{\left\{\left(|\partial(M s) / \partial x|^{2}\right)+\left(\partial^{2}(M s) / \partial x^{2}\right)^{2}\right\}}{K^{2}}
$$

At the peak, the gradient modulus is zero, while the second derivative is always a local maximum. Therefore, the diffusion coefficient at the peak is relatively small and the diffusion is slower, which is conducive to maintaining details such as peaks and boundaries.

To sum up, the essence of the morphological Haar wavelet Perona-Malik filter model is that the morphological Haar wavelet is utilized to presmooth the noisy signal firstly before calculating the diffusion coefficient; then, the gradient and the second derivative on the presmoothed signal can be computed to obtain the diffusion coefficient, which makes the diffusion process not only denoise effectively but also retain the meaningful details as much as possible.

\section{Adaptive Morphological Wavelet Perona-Malik Filtering Algorithm}

The simulation analysis shows that the morphological wavelet Perona-Malik filter model still has the limitations of identifying strong noise points and boundaries in the signal. At the strong noise point, even if the morphological wavelet is used for prefiltering, the signal gradient is still relatively large. Subsequently, the diffusion coefficient at the strong noise point is relatively small, the diffusion is relatively weak, and even reverse diffusion exists; thus, the noise will be enlarged instead of being removed. Therefore, a strong noise point recognition operator is designed to adaptively recognize strong noise points, and the same direction average gradient is used to adaptively remove strong noise points.

4.1. Strong Noise Point Recognition Operator. In order to identify whether a point is a strong noise point, a strong noise point recognition operator is constructed. Firstly, the mean and variance of the strong noise points are calculated as

$$
\begin{aligned}
& m_{i}=\frac{1}{2}\left(u_{i-1}+u_{i+1}\right), \\
& \sigma_{i}^{2}=\left|\frac{1}{2}\left(u_{i-1}^{2}+u_{i+1}^{2}\right)-m_{i}^{2}\right|,
\end{aligned}
$$

where $u_{i}$ is the amplitude at the data point $i$.

Secondly, a strong noise point recognition operator is constructed as

$$
\lambda_{i}=\frac{\left|u_{i}-m_{i}\right|^{2}}{\sigma_{i}^{2}} .
$$

If $\lambda_{i}<1$ and the noise energy is less than the signal energy, the data point is a normal signal and should be retained; if $\lambda_{i} \geq 1$ and the noise energy is greater than the 
signal energy, the data point is a strong noise point and should be diffused.

4.2. Average Gradient in the Same Direction. In order to solve the problem of large gradient value at the strong noise point, the gradient value of the strong noise point can be replaced by the two-step average gradient value at the left and right directions of the strong noise point. Consequently, the gradient value of the strong noise point can be reduced to achieve the purpose of effectively removing strong noise points.

Suppose the amplitude at the noise point $i$ is $u_{i}$; then, the average gradient values $\nabla L_{i}^{\text {ave }}$ and $\nabla R_{i}^{\text {ave }}$ of the noise point $i$ in the left and right directions in the same direction are, respectively, as follows:

$$
\begin{aligned}
\nabla L_{i}^{\mathrm{ave}} & =\frac{\left(\left|u_{i-2}-u_{i-1}\right|+\left|u_{i-1}-u_{i}\right|\right)}{2}, \\
\nabla R_{i}^{\mathrm{ave}} & =\frac{\left(\left|u_{i+2}-u_{i+1}\right|+\left|u_{i+1}-u_{i}\right|\right)}{2} .
\end{aligned}
$$

The average of the two gradients in the same direction is used to replace the gradients in the left and right directions of noise points $i$, which ensures that the diffusion of noise points $i$ is opposite and can better maintain the peaks and boundaries. Generally, the probability that the left and right points $i-2, i-1, i+1$, and $i+2$ are noise points is relatively low while the data point $i$ is a noise point. Therefore, the average of the two gradients in the left and right directions of the data point $i$ can reduce the gradient value of the isolated strong noise point, which facilitates forward diffusion denoising.

Then,

$$
\left|\frac{\partial(M u)}{\partial x}\right|=\left|\nabla L_{i}^{\text {ave }}\right|+\left|\nabla R_{i}^{\text {ave }}\right|
$$

Generally, the gradient value of strong Gaussian noise is relatively large; then, the adaptive Perona-Malik model based on the average gradient in the same direction can reduce the gradient value of the strong noise point relatively, which is beneficial to the subsequent forward diffusion.

4.3. The Discrete Format of the Improved Model. The discrete calculation format of the improved adaptive morphological wavelet Perona-Malik filtering algorithm is presented as

$$
u_{i}^{k+1}=u_{i}^{k}+\Delta t \cdot\left(C_{L} \cdot D_{L} u_{i}^{k}+C_{R} \cdot D_{R} u_{i}^{k}\right),
$$

where

$$
\begin{aligned}
D_{R} u_{i}^{k} & =u_{i+1}^{k}-u_{i}^{k}, \\
\bar{u}^{k} & =\mathrm{Mu}^{k}, \\
D_{L} \bar{u}_{i}^{k} & =\bar{u}_{i-1}^{k}-\bar{u}_{i}^{k}, D_{R} \bar{u}_{i}^{k}=\bar{u}_{i+1}^{k}-\bar{u}_{i}^{k},
\end{aligned}
$$

$$
\begin{aligned}
a_{i}^{k} & =\left(\frac{\partial^{2} \bar{u}^{k}}{\partial x^{2}}\right)_{i}^{2} \\
\left(\frac{\partial^{2} \bar{u}^{k}}{\partial x^{2}}\right)_{i} & =\left(\bar{u}^{k}\right)_{i-1}-2\left(\bar{u}^{k}\right)_{i}+\left(\bar{u}^{k}\right)_{i+1} .
\end{aligned}
$$

If $\lambda \geq 1$,

$$
\begin{aligned}
& C_{L}=\tilde{g}\left(\nabla L_{i}^{\mathrm{ave} 2}+a_{i}^{k}\right), \\
& C_{R}=\tilde{g}\left(\nabla R_{i}^{\mathrm{ave} 2}+a_{i}^{k}\right) .
\end{aligned}
$$

Otherwise,

$$
\begin{aligned}
& C_{L}=\tilde{g}\left(\left|D_{L} \bar{u}_{i}^{k}\right|^{2}+a_{i}^{k}\right), \\
& C_{R}=\widetilde{g}\left(\left|D_{R} \bar{u}_{i}^{k}\right|^{2}+a_{i}^{k}\right) .
\end{aligned}
$$

$\widetilde{g}(s)=g\left(s^{1 / 2}\right), k$ is the number of iterations, and $\Delta t$ is the time step that satisfies $0 \leq \Delta t \leq 1 / 4$. This is the condition for calculating the stability of the format. It can be proven easily that $m \leq u_{i}^{k+1} \leq M$, where $m=\min \left\{u_{i-1}^{k}, u_{i}^{k}, u_{i+1}^{k}\right\}$ and $M=\max \left\{u_{i-1}^{k}, u_{i}^{k}, u_{i+1}^{k}\right\}$, that is, the signal after denoising will not generate new extreme points.

4.4. Steps of the Improved Algorithm. According to the aforementioned improved algorithm and its discrete calculation format, the steps of the improved algorithm for denoising rolling bearing fault vibration signals can be presented as follows.

Step 1. Set the parameters such as the gradient threshold parameter $K$, time step $\Delta t$, and the number of iterations $I$.

Step 2. Let the number of iterations $k=1$ and the signal to be diffused $u^{k-1}$ be the noisy signal $u_{0}$.

Step 3. According to equation (12), calculate the left and right gradients $D_{L} u^{k-1}$ and $D_{R} u^{k-1}$ of the signal to be diffused $u^{k-1}$.

Step 4. Use the morphological Haar wavelet to presmooth the signal to be diffused $u^{k-1}$, and calculate the left gradient $D_{L} \bar{u}^{k-1}$, the right gradient $D_{R} \bar{u}^{k-1}$, and the second derivative $a^{k-1}$ of the presmoothed signal $\bar{u}^{k-1}$ based on equations (13) (15).

Step 5. According to equation (9), calculate the value of the strong noise point identification operator $\lambda_{i}$ at each point of the presmoothed signal $\bar{u}^{k-1}$. Combined with equation (10), calculate the two-step average gradient in the left and right directions $\nabla L^{\text {ave }}$ and $\nabla R^{\text {ave }}$ of the presmoothed signal $\bar{u}^{k-1}$.

Step 6. If $\lambda \geq 1$, calculate the left-right diffusion coefficient $C_{L}$ and $C_{R}$ using equation (16); otherwise, calculate the leftright diffusion coefficient $C_{L}$ and $C_{R}$ using equation (17).

Step 7. Combined with equation (11), calculate the diffusion signal $u^{k}$ of the iteration $k$. 

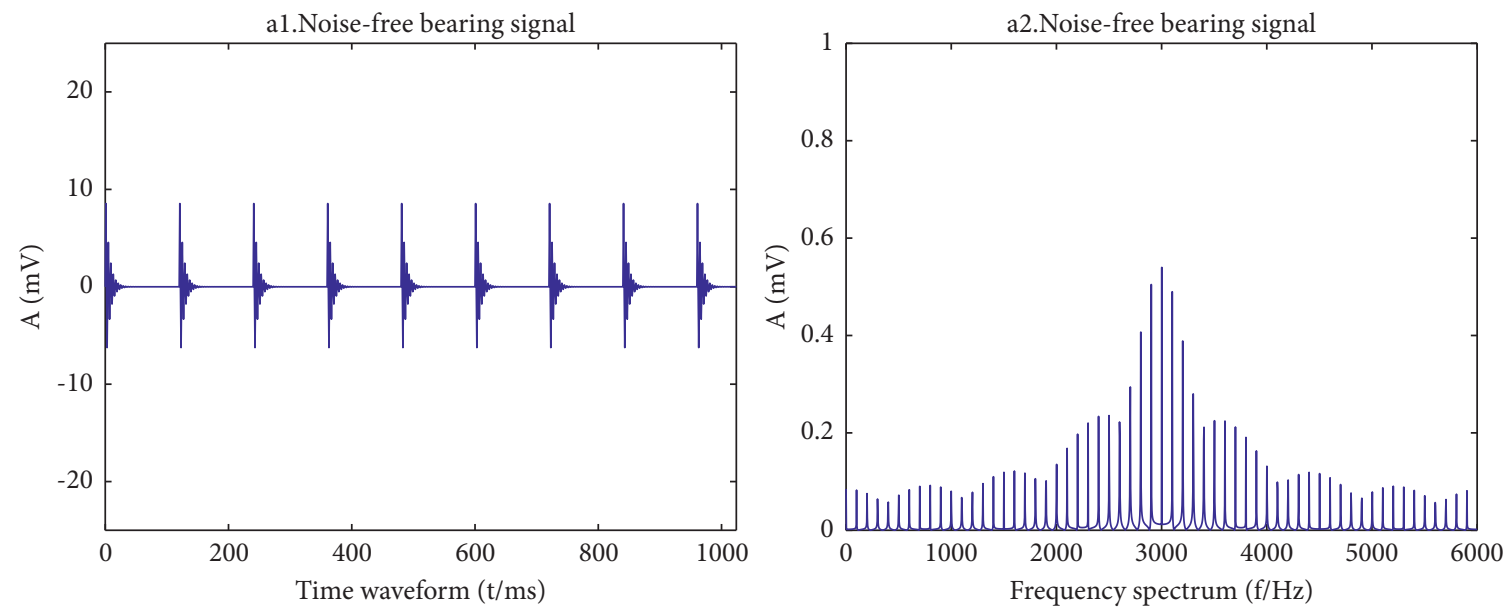

(a)

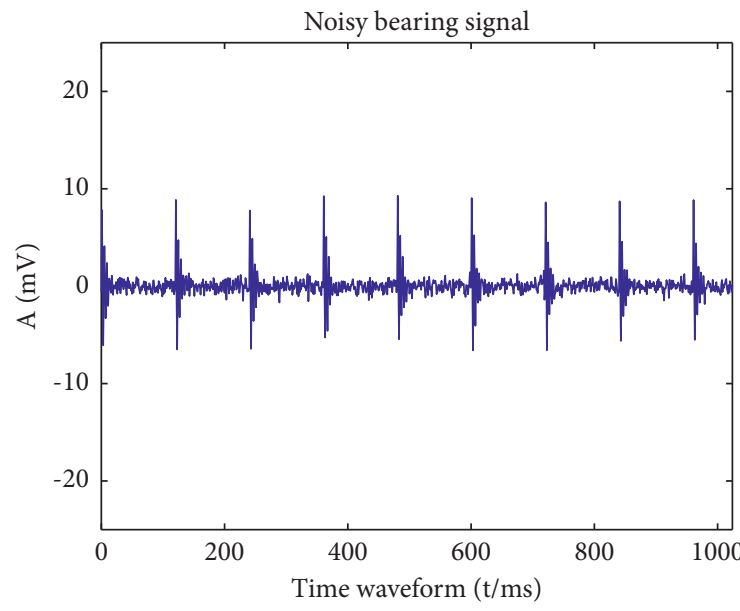

(b)

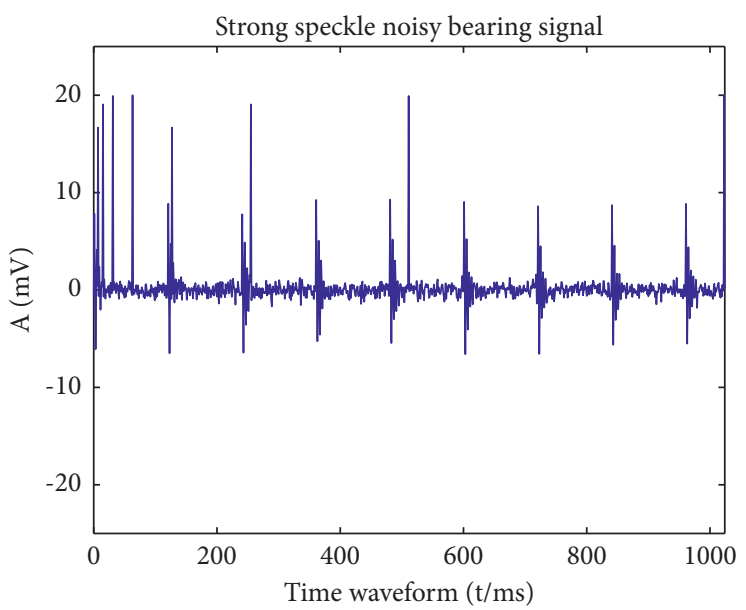

(c)

FIGURE 1: Simulation signal of rolling bearing. (a) Noisy-free bearing signal. (b) Noisy bearing signal. (c) Strong speckle noisy bearing signal.

Step 8. If $k=I$, return to Step 9; if $k<I$, then let $k=k+1$, return to Step 3.

Step 9. Output the denoising signal $u^{k}$.

\section{Rolling Bearing Experiment}

5.1. Simulation Signal Analysis. The simulation signals of rolling bearings are used to compare the denoising performance of the wavelet threshold denoising method, morphological wavelet method, Perona-Malik model, Catté model, and the proposed algorithm to demonstrate the effectiveness of the proposed algorithm.

Assume that the vibration simulation signal of a singlepoint damage fault on the outer ring of rolling bearing is presented as

$$
y(t)=y_{0} e^{-\xi \omega_{n} t} \sin \omega_{n} \sqrt{1-\xi^{2}} t
$$

where the natural frequency of the attenuated vibration $f_{n}=3000 \mathrm{~Hz}$, the natural angular frequency of the vibration $\omega_{n}=2 \pi f_{n}$, the displacement constant $y_{0}=10$, the damping coefficient $\xi=0.1$, the sampling frequency $f_{s}=12000 \mathrm{~Hz}$, and the number of sampling points is 4096, as shown in Figure 1(a) (Figure a1 represents the time-domain waveform and Figure a2 represents the frequency spectrum).

The rolling bearing fault signal plus Gaussian white noise with the standard deviation $\sigma=1$ is the noisy signal to be analyzed, as shown in Figure 1(b). In order to simulate the isolated strong noise points, the intensity (amplitude) of some noise points is enhanced, as shown in Figure 1(c).

The simulation experiment can be divided into three parts, i.e., (1) comparing the denoising performance difference between the Perona-Malik model and the classic methods (such as wavelet soft threshold denoising method and morphological Haar wavelet method) to indicate the superiority of the nonlinear anisotropic diffusion filtering method, as shown in Figure 2, (2) comparing the denoising performance of the Perona-Malik model, the Catté model, and the morphological 

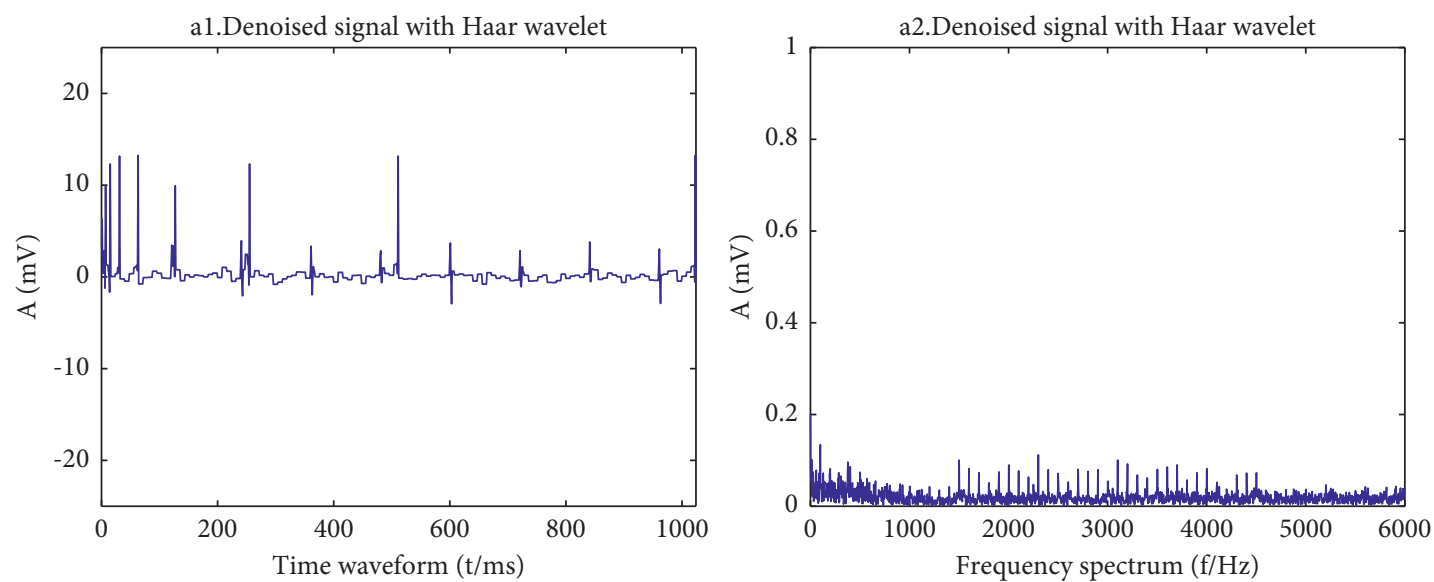

(a)
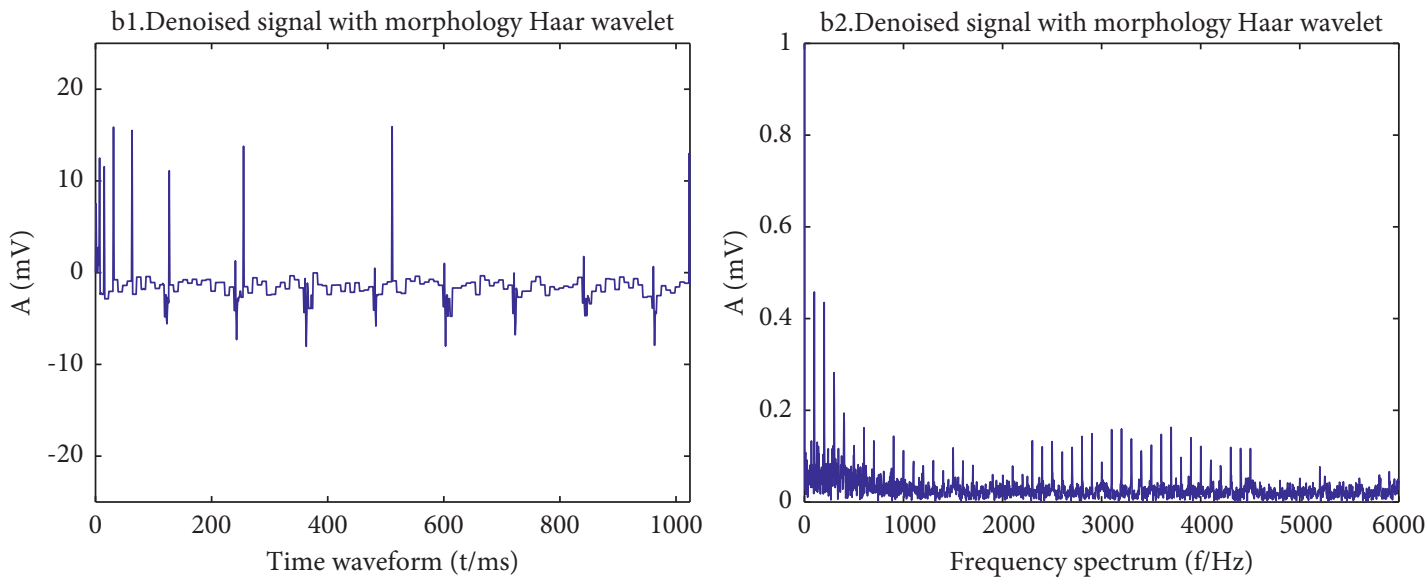

(b)
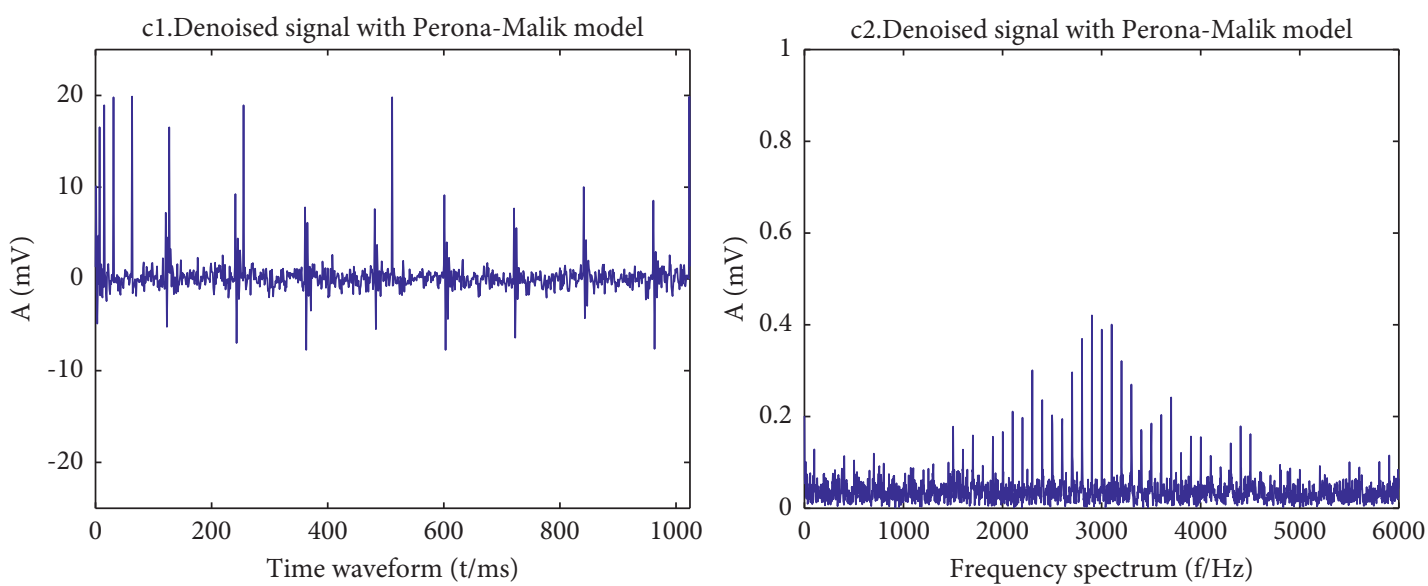

(c)

FIGURE 2: Comparison between PDE model and other denoising algorithms. (a) Denoised signal with Haar wavelet. (b) Denoised signal with morphology Haar wavelet. (c) Denoised signal with the Perona-Malik model.

Haar wavelet Perona-Malik model (using its own gradient) to illustrate the necessity of introducing the morphological Haar wavelet and the second derivative, as shown in Figure 3, and (3) checking whether the same direction average gradient denoising performance should be utilized to indicate that the same direction average gradient can effectively filter out strong noise points adaptively, as shown in Figure 4.
Figure 2 shows that the classic denoising method filters out useful fault characteristic information at the same time while denoising. Although the wavelet soft threshold denoising and morphological Haar wavelet methods filter out noise, a lot of useful information is also filtered out (as shown in Figures 2(a) and 2(b)). It is because their denoising effect is closely related to the selection of threshold value. As some isolated strong noise 

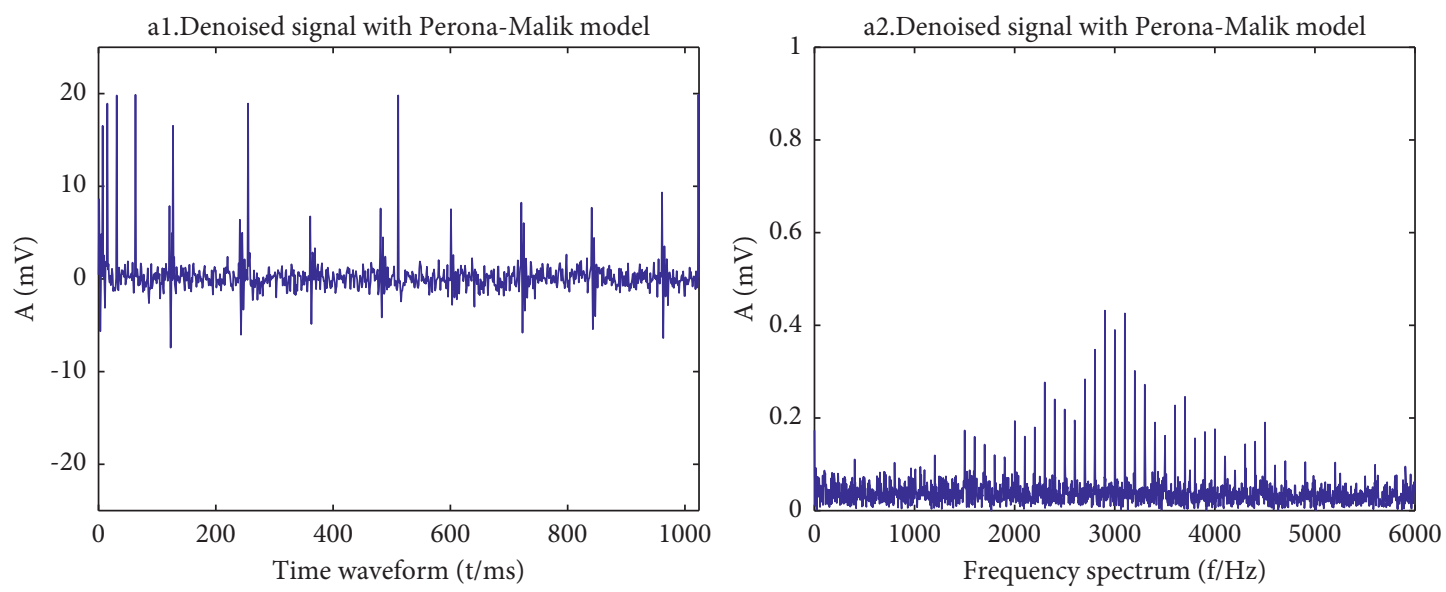

(a)
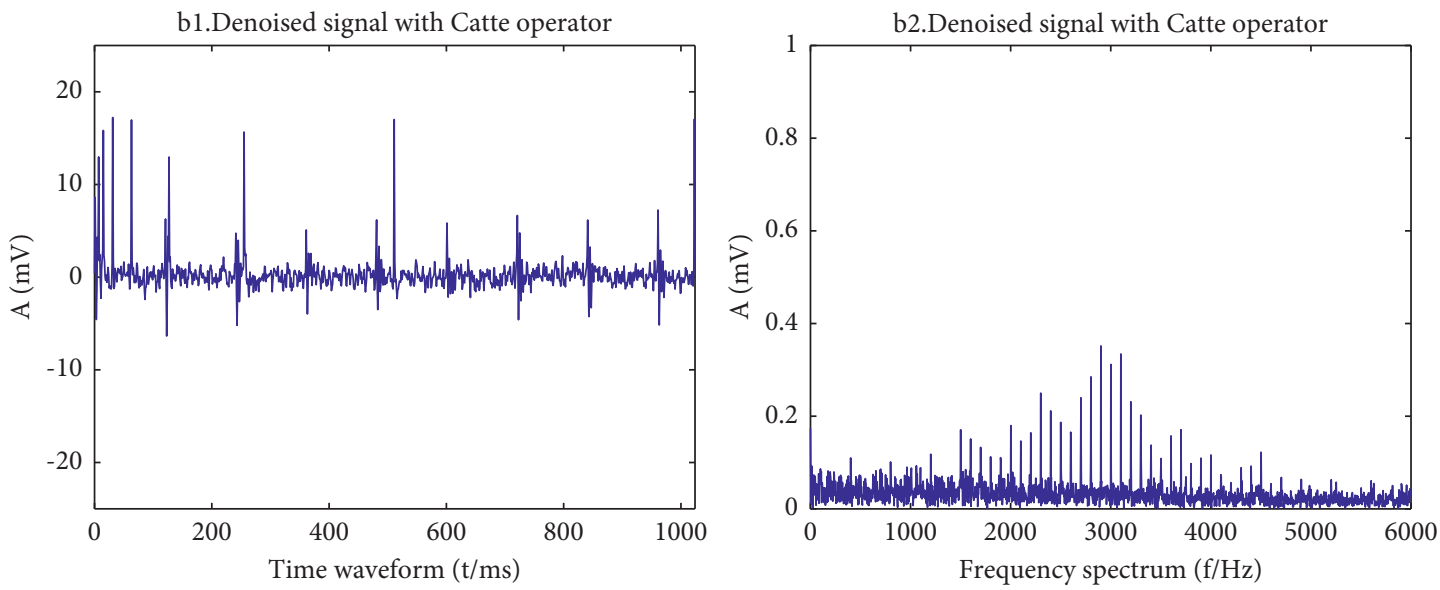

(b)
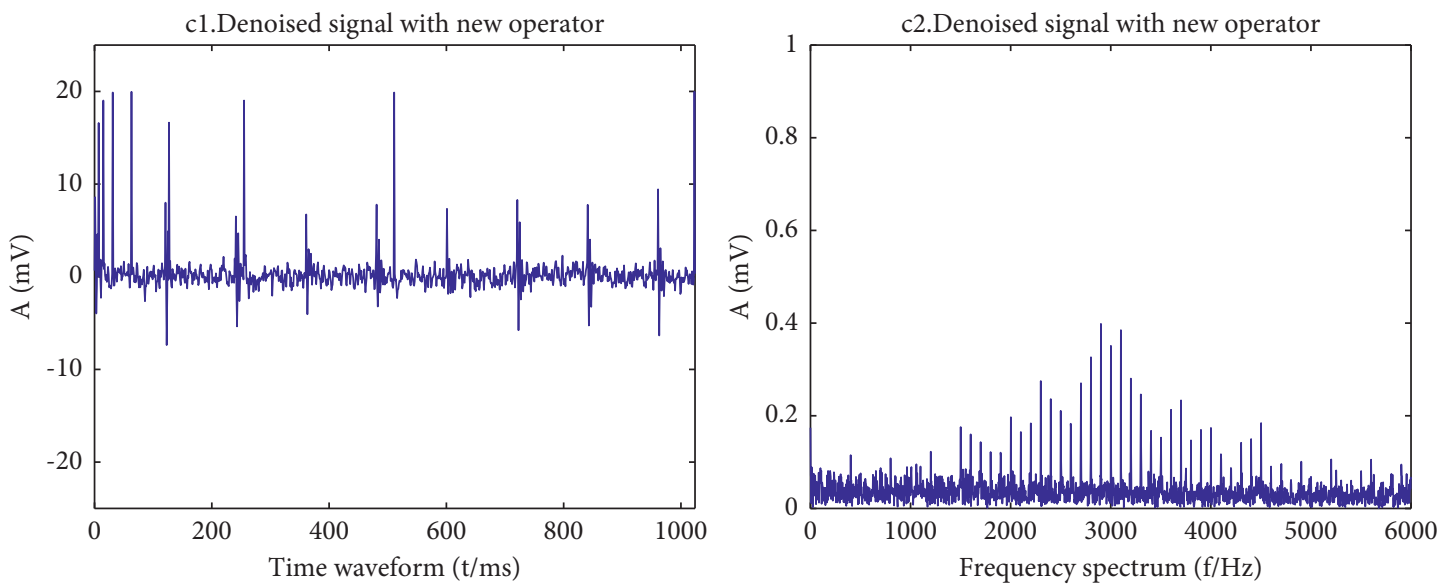

(c)

Figure 3: Comparison between PDE model and its improved models. (a) Denoised signal with Perona-Malik model. (b) Denoised signal with Catté operator. (c) Denoised signal with new operator.

points are added to the signal, the wavelet decomposition coefficient of the high-frequency part is too large, which affects the selection of global threshold value. Therefore, a lot of useful high-frequency information is filtered out as noise.

Figure 2 also shows that the classical denoising methods also filter out useful fault characteristic information. Although the wavelet soft threshold denoising and morphological Haar wavelet denoising methods filter out noise, a large amount of useful information is also filtered out (as shown in a2 and b2). This is because their denoising effect is closely related to the selection of threshold value. As some isolated strong noise points are added to the signal, the wavelet decomposition 

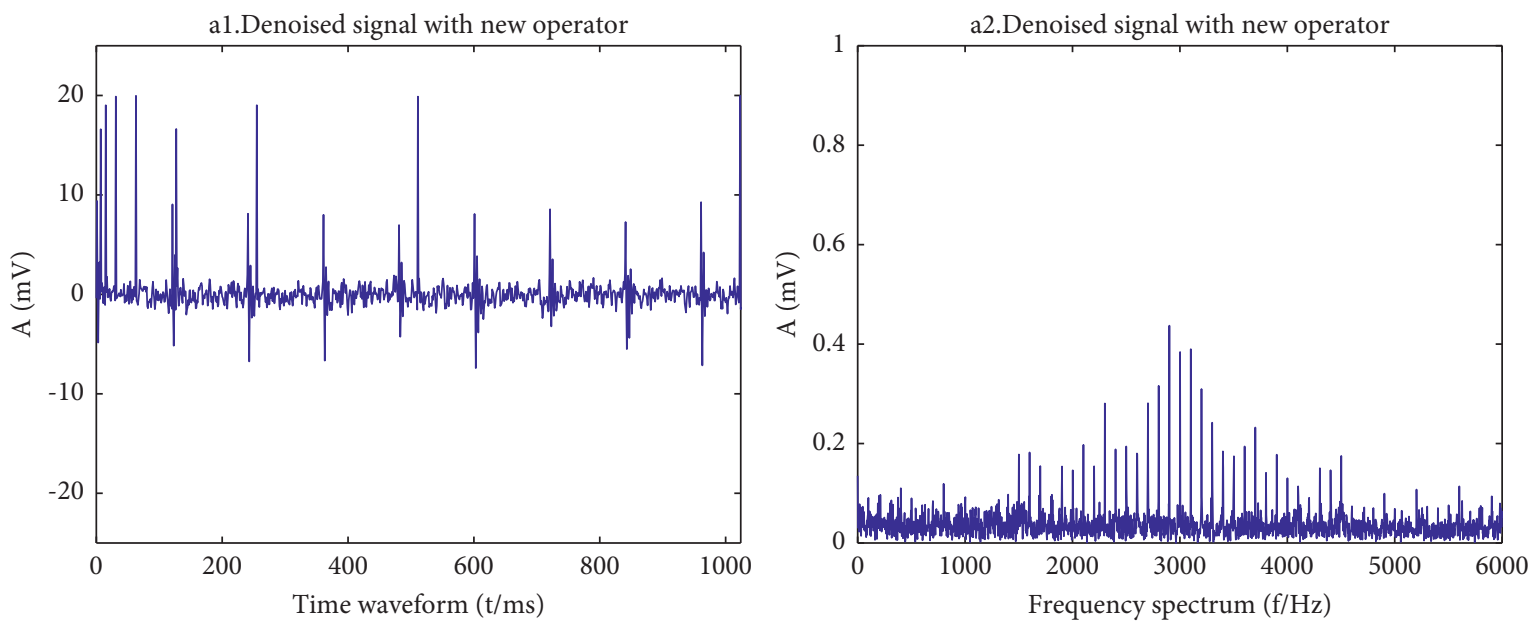

(a)
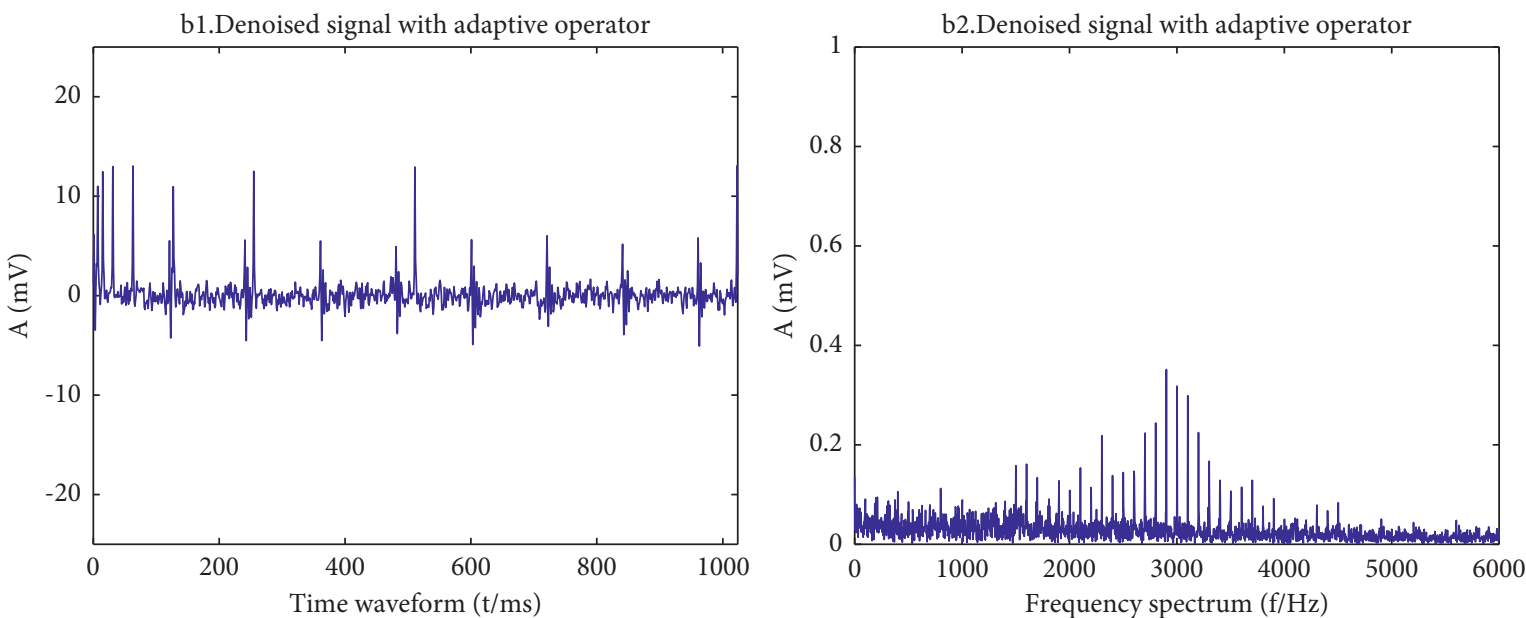

(b)

FIGURE 4: Comparison between original gradient and its average gradient. (a) Denoised signal with new operator. (b) Denoised signal with adaptive operator.

TABLE 1: SNR of several denoising algorithms.

\begin{tabular}{lcccc}
\hline Gaussian white noise standard deviation $\sigma$ & $\begin{array}{c}\text { Perona-Malik } \\
\text { model }\end{array}$ & $\begin{array}{c}\text { Catté } \\
\text { model }\end{array}$ & $\begin{array}{c}\text { The improved model (own gradient) } \\
\text { (average gradient) }\end{array}$ & $\begin{array}{c}\text { The improved model } \\
\text { (averager }\end{array}$ \\
\hline 0.2 & 1.64 & 1.65 & 1.71 & 3.05 \\
0.5 & 0.86 & 1.09 & 1.18 & 2.39 \\
1 & -1.14 & -0.43 & -0.23 & 0.71 \\
2.5 & -6.60 & -5.98 & -4.85 & -4.25 \\
\hline
\end{tabular}

coefficient of the high-frequency part is too large, which affects the selection of global threshold value. Therefore, a lot of useful high-frequency information is filtered out as noise.

The Perona-Malik nonlinear anisotropic diffusion filter model not only effectively filters out noise but also maintains the fault characteristic information, which is different from the above methods (as shown in Figure 2(c)).

As shown in Figure 3, the Perona-Malik model, the Catté model, and the improved morphological Haar wavelet Perona-Malik model (with its own gradient) all take into account denoising and feature retention, indicating that the PDE method has strong local adaptability, edge preservation, and noise reduction and high flexibility.

As shown in Figure 3, the Perona-Malik model, Catté model, and the improved morphological Haar wavelet Perona-Malik model (with its own gradient) all give good consideration to denoising and feature retention, indicating that the PDE method has strong local adaptability, edge preservation, and noise reduction and high flexibility. At the same time, due to the nonlinearity of the morphological Haar 

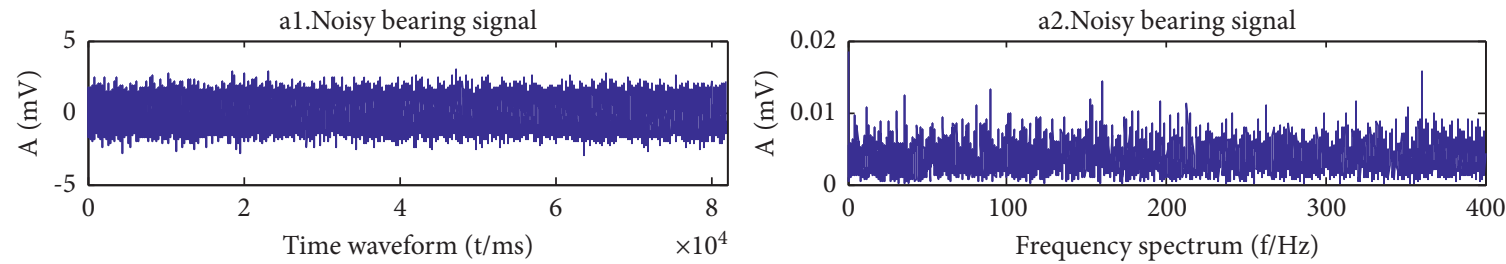

(a)
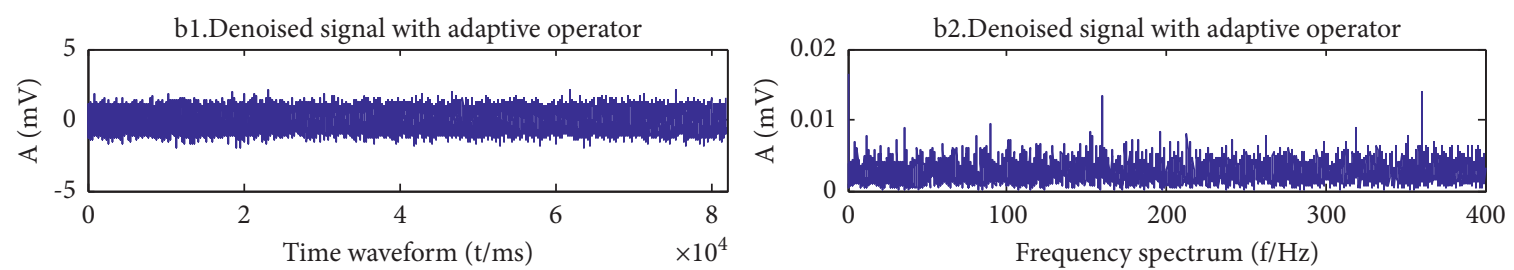

(b)
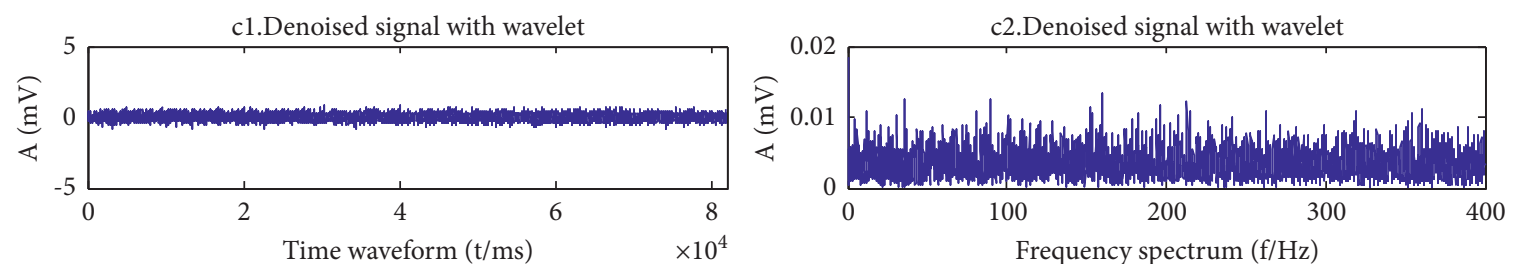

(c)
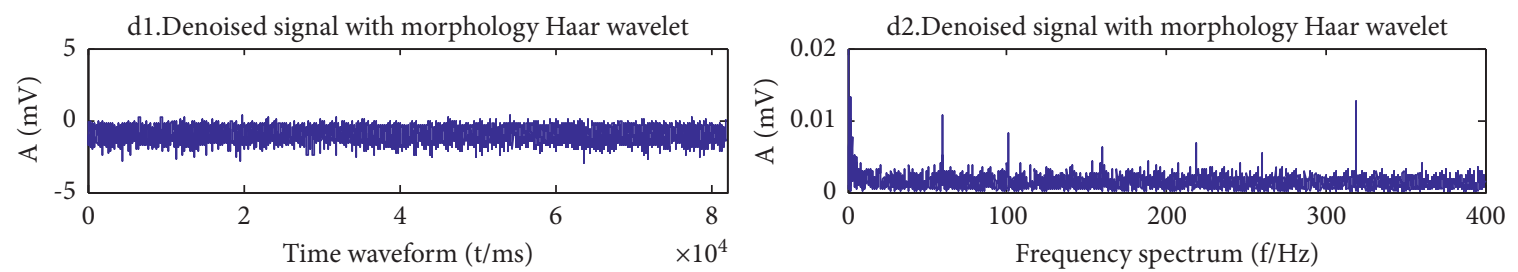

(d)
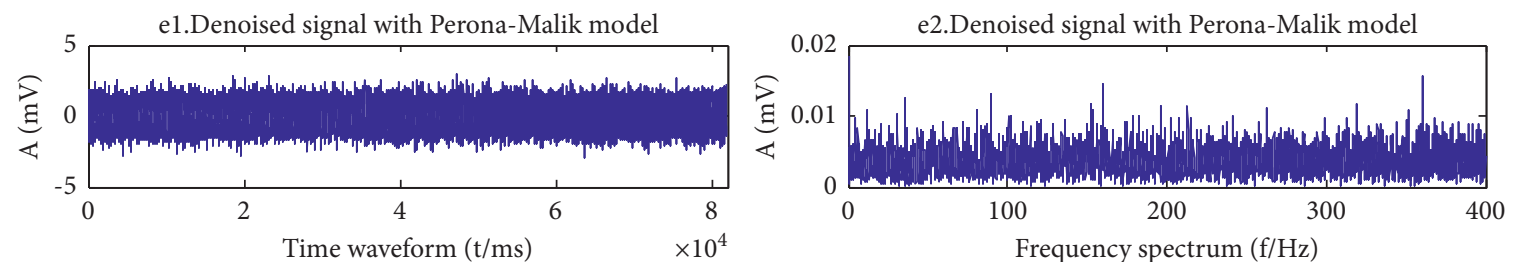

(e)

Figure 5: CWRU measured data. (a) Noisy bearing signal. (b) Denoised signal with adaptive operator. (c) Denoised signal with wavelet. (d) Denoised signal with morphology Haar wavelet. (e) Denoised signal with Perona-Malik model.

wavelet, the improved model has better denoising performance, so the ability to maintain fault features is better, and the peak information in the signal is well preserved.

At the same time, due to the nonlinearity of the morphological Haar wavelet, the denoising performance and the fault feature retention ability of the improved model are better, and the peak information in the signal can be maintained effectively. The conclusion can be confirmed by the SNR of each algorithm, as presented in Table 1. The SNR of the improved model is higher under different standard deviations of Gaussian white noise, which indicates that the introduction of the morphological Haar wavelet and the second derivative into the Perona-Malik model is necessary, and its filtering performance has been effectively improved.
Figures 4(a) and 4(b) show that the gradient of the strong noise point itself and the same direction average gradient of the strong noise point are utilized to improve the diffusion coefficient of the denoising results, respectively. From the signal-to-noise ratio (SNR), as presented in Table 1, we can see that the same direction average gradient of the strong noise makes the strong noise point value less, which can effectively suppress the reverse diffusion of strong noise points. The signal-to-noise ratio of the improved model was improved (signal-to-noise ratio increased by about $1 \mathrm{~dB}$ ), and strong noise adaptive filtering was realized.

As shown in Figures 4(a) and 4(b), respectively, with gradient and high noise point itself with strong noise point average gradient direction to improve the diffusion 

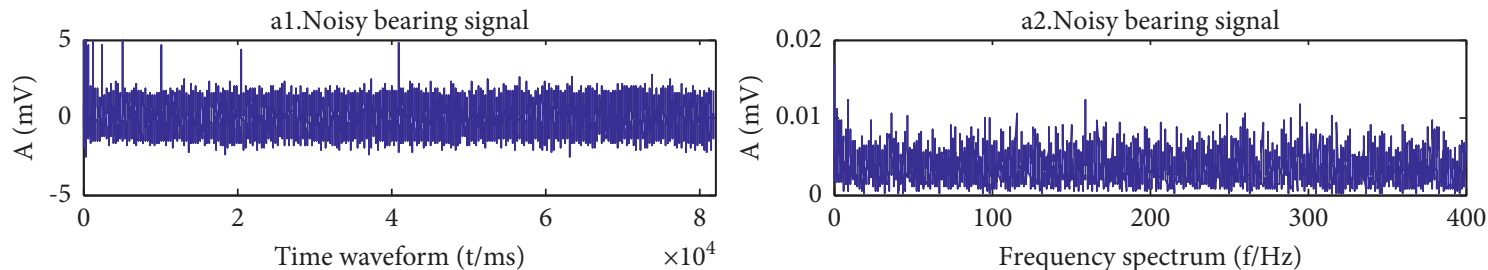

(a)
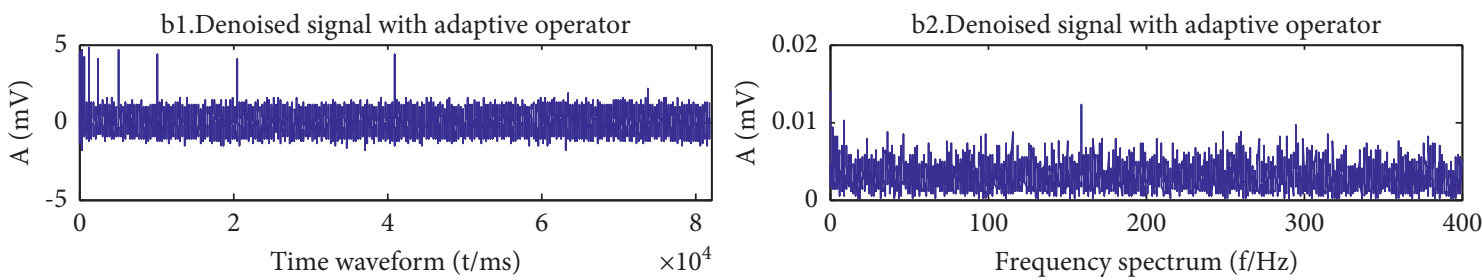

(b)
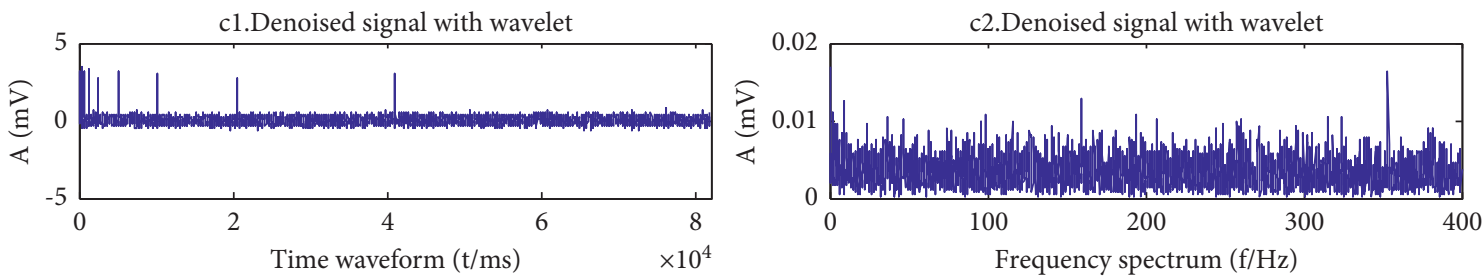

(c)
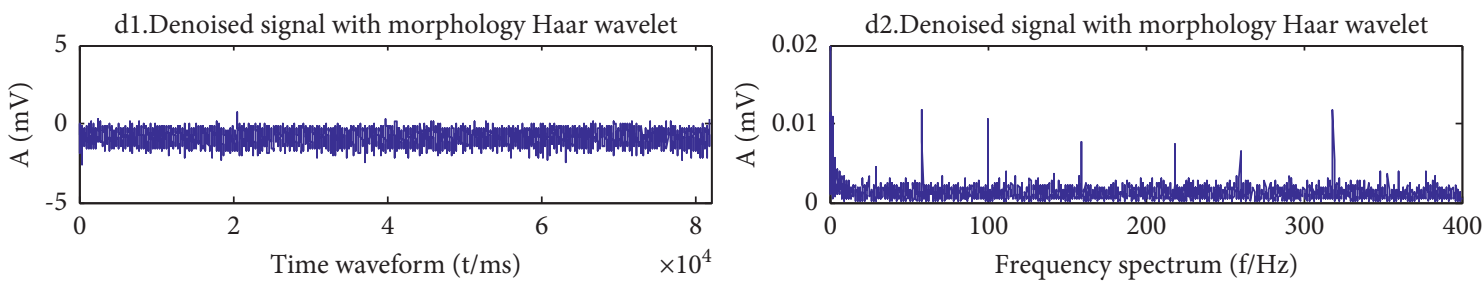

(d)
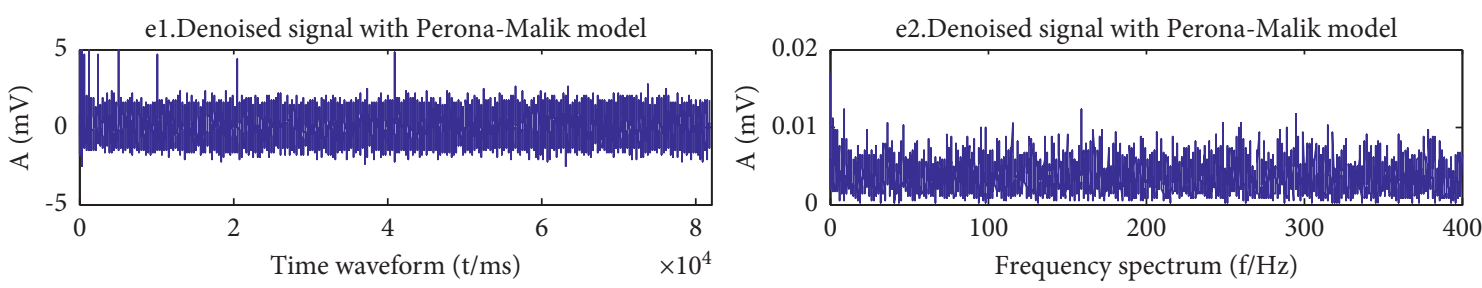

(e)

Figure 6: CWRU measured data. (a) Noisy bearing signal. (b) Denoised signal with adaptive operator. (c) Denoised signal with wavelet. (d) Denoised signal with morphology Haar wavelet. (e) Denoised signal with Perona-Malik model.

coefficient of the denoising results, for its signal-to-noise ratio (SNR) as presented in Table 1, the experimental results show that a higher average gradient algorithm with strong noise makes the strong noise gradient value less, which can effectively suppress strong noise point reverse diffusion. The signal-to-noise ratio (SNR) of the improved model was improved by about $1 \mathrm{~dB}$, and strong noise adaptive filtering was realized.

5.2. Measured Signal Analysis. The experimental data of the Electrical Engineering Laboratory of Case Western Reserve University (CWRU) in the United States are used to be the measured vibration signal of rolling bearing. Figure 5 (Figure al indicates the time-domain waveform and Figure a2 indicates the frequency spectrum) shows the signal derived from the fault vibration data of the inner ring of the motor end bearing, the sampling frequency is $12 \mathrm{kHz}$, the shaft speed is $1772 \mathrm{rpm}$, the fault diameter is $0.1778 \mathrm{~mm}$, and the fault characteristic frequency can be calculated as $159.9289 \mathrm{~Hz}$ according to the empirical formula. Due to the excellent experimental conditions and environment, it is easy to obtain the fault characteristic frequency by analyzing the data directly. In order to increase the difficulty of the experiment and simulate a more real mechanical operating environment, noise signals are added to the experimental 
data and then denoised in this paper's experiment. In the first part, the analog white noise signal with the amplitude of 0.6 is directly added, and in the second part, the analog white noise signal with the amplitude of 0.5 and several pulse signals are added. Then, the method proposed in this paper and other methods are used for denoising before comparing the spectrum analysis results, as shown in Figures 5 and6, respectively.

The improved model is used to remove the noise in the signal, as shown in Figures 5(a) and 6(a), and the denoised signal is shown in Figures 5(b) and 6(b) (Figure b1 represents the time-domain waveform and Figure b2 represents the frequency spectrum). In order to further compare the denoising performance of the proposed method with that of the classical wavelet method,the morphological wavelet Perona-Malik model, the wavelet soft threshold denoising method, the morphological wavelet method, and the Perona-Malik model are introduced to remove the noise in the signal, as shown in Figures 5(a) and 6(a), respectively, and the denoising signal is presented as shown in Figures 5(c)-5(e) and 6(c)-6(e) (c1, d1, and e1 represent the time-domain waveforms, and $\mathrm{c} 2, \mathrm{~d} 2$, and $\mathrm{e} 2$ represent the frequency spectrum).

The measured signal experiments in Figures 5 and 6 show that although the Perona-Malik model retains the details of the signal, it does not effectively remove the noise in the signal. The effect of the soft threshold wavelet denoising method and the morphological wavelet denoising method is not ideal; the addition of an isolated strong noise point affects the selection of threshold value, these two methods will not only denoise the signal at the same time but also remove the useful details of the signal and even make the signal after denoising distortion.

In contrast, the proposed model can preserve the details of the signal and remove the noise in the signal effectively and highlight its characteristic frequency (the characteristic frequency of the inner ring fault is $159 \mathrm{~Hz}$ ), which creates conditions for the extraction of weak fault characteristics with a strong noise background. Further experiments also show that the improved model is also very effective for denoising the fault signals of bearing outer rings, rolling elements, and other mechanical parts such as gears. Therefore, the improved model can be used for denoising mechanical vibration signals, which can denoise and preserve signal characteristics.

\section{Conclusions}

The traditional Perona-Malik model has a relatively largenoise level, and the denoising effect is not ideal, especially when the signal is interfered by impulsive noise. In order to deal with these problems, the morphological Haar wavelet transform is introduced to preprocess the noisy signal; then, the first and second derivatives are utilized to construct a novel high-order diffusion coefficient function to suppress. A strong noise point recognition operator is designed, and the same two-step average gradient of the strong noise point is used to replace the gradient of the noise point itself, which can suppress the back diffusion of the strong noise point and denoise adaptively better. The denoising results of the simulation signal and the vibration signal of the rolling bearing fault experiment verify the feasibility and effectiveness of the algorithm in this paper.

In the practice of denoising, the two parameters of the gradient threshold and the iteration number of the improved model are obtained through repeated experiments, which makes the two parameters may not be optimal. Therefore, the adaptive values of gradient threshold and the iteration number of the improved model need to be further studied, which is one of the research directions in the future.

\section{Data Availability}

No data were used to support this study.

\section{Conflicts of Interest}

The authors declare that they have no conflicts of interest.

\section{Acknowledgments}

This work was supported by the Science and Technology Project of Sichuan Province, No. 2019YJ0266, the theory and method of complex equipment fault diagnosis based on Stransform.

\section{References}

[1] Z. K. Peng and F. L. Chu, "Application of the wavelet transform in machine condition monitoring and fault diagnostics: a review with bibliography," Mechanical Systems and Signal Processing, vol. 18, no. 2, pp. 199-221, 2004.

[2] H. Li, Y. Zhou, and F. Tian, "Wavelet-based vibration signal de-noising algorithm with a new adaptive threshpld function," Chinese Journal of Scientific Instrument, vol. 36, no. 10, pp. 2200-2206, 2015.

[3] T. Schreiber, "Extremely simple nonlinear noise-reduction method," Physical Review, vol. 47, no. 4, pp. 2401-2404, 1993.

[4] J. Yang, X. U. Jinwu, D. Yang, and L. I. Min, "Noise reduction method for nonlinear time series based on principal manifold learning and its application to fault diagnosis," Journal of Mechanical Engineering, vol. 42, no. 8, 2005.

[5] B. Guangqing, C. Yong, and Y. Guojin, "De-noising of rolling bearing fault vibration signal based on empirical mode decomposition threshold," Computer Engineering and Applications, vol. 51, no. 10, pp. 205-210, 2015.

[6] J. M. Malik and P. Perona, "Scale-space and edge detection using anisotropic diffusion'," IEEE Transactions on Pattern Analysis and Machine Intelligence, vol. 12, no. 7, 1990.

[7] Z. Ren, C. He, and Q. Zhang, "Fractional order total variation regularization for image super-resolution," Signal Processing, vol. 93, no. 9, pp. 2408-2421, 2013.

[8] D. Jiang, X. Feng, and G. Song, "An anisotropic diffusion equation based on nonlinear wavelet shrinkage," Acta Electronica Sinica, vol. 34, no. 1, pp. 170-172, 2006.

[9] J. Bai and X.-C. Feng, "Fractional-order anisotropic diffusion for image denoising," IEEE Transactions on Image Processing, vol. 16, no. 10, pp. 2492-2502, 2007.

[10] L. Yong and J. Liu, "An improved anisotropic diffusion of cattle follicle ultrasound images de-noising algorithm," in Proceedings of the Intelligent Computing Methodologies, pp. 418-430, Wuhan, China, Auguest 2018. 
[11] L. Feng and J. Liu, "Anisotropic diffusion for image denoising based on diffusion tensors," Journal of Visual Communication and Image Representation, vol. 23, no. 3, pp. 516-521, 2012.

[12] Z. Xiao, Z. Xu, F. Zhang et al., "ESPI filtering method based on anisotropic coherence diffusion and Perona-Malik diffusion," Chinese Optics Letters, vol. 11, no. 10, 2013.

[13] J. Yuan and J. Wang, "Perona-malik model with a new diffusion coefficient for image denoising," International Journal of Image and Graphics, vol. 16, no. 2, 2016.

[14] V. Kamalaveni, R. A. Rajalakshmi, and K. A. Narayanankutty, "Image denoising using variations of perona-malik model with different edge stopping functions," Procedia Computer Science, vol. 58, pp. 673-682, 2015.

[15] N. Ally, J. Nombo, K. Ibwe, A. T. Abdalla, and B. J. Maiseli, "Diffusion-driven image denoising model with texture preservation capabilities," Journal of Signal Processing Systems, vol. 6, 2021.

[16] M. Vera, E. Gonzalez, Y. Huérfano, E. Gelvez, and O. Valbuena, "New anisotropic diffusion operator in images filtering," Journal of Physics: Conference Series, vol. 1448, no. 1, Article ID 012019, 2020.

[17] E. Cuevas, H. Becerra, and A. Luque, "Anisotropic diffusion filtering through multi-objective optimization," Mathematics and Computers in Simulation, vol. 181, 2021.

[18] W. Wu and X. Chen, "Strong noise signal filtering algorithm based on wavelet transform module and modified PeronaMalik model," Journal of Vibration and Shock, vol. 37, no. 17, pp. 277-282, 2018.

[19] I. M. Hamdani, S. Anam, and N. Shofianah, "Counting of bacterial colonies of the low quality image using perona-malik diffusion filters and image morphology operators," Journal of Physics Conference, vol. 1490, no. 1, Article ID 012058, 2020.

[20] H. Wu, A. Yin, and S. Qin, "Vibration signal denoising based on PDE," Journal of Mechanical Engineering, vol. 45, no. 5, pp. 91-94, 2009.

[21] A. Yin, H. Wu, and S. Qin, "Principle and method of PDE filtering," Journal of Vibration and Shock, vol. 29, no. 10, pp. 232-235+262, 2010.

[22] A. Yin, L. Sun, and J. Wang, "Application of partial differential equation in the purification of axial locus," Journal of Chongqing University, vol. 34, no. 12, pp. 72-77, 2011.

[23] Y. Xu, Q. Huang, and Y. Fang, "An adaptive denoising method for vehicle acceleration signal based on partial differential equation," Chinese Journal of Sensors and Actuators, vol. 22, no. 11, pp. 1606-1611, 2009.

[24] H. Lan, Y. Gao, and H. Ye, "Suppression of white noise in partial discharge signal by partial differential equation," High Voltage Apparatus, vol. 49, no. 4, pp. 43-48, 2013.

[25] L. Alvarez, P.-L. Lions, and J.-M. Morel, "Image selective smoothing and edge detection by nonlinear diffusion. II," SIAM Journal on Numerical Analysis, vol. 29, no. 3, pp. 845-866, 1992.

[26] C. Francine, L. Pierre Louis, M. Jean Morel, and C. Tomeu, "Image selective smoothing and edge detection by nonlinear diffusion," SIAM Journal on Numerical Analysis, vol. 29, no. 1, pp. 182-193, 1992.

[27] J. Chan, H. Ma, T. Saha, and C. Ekanayake, "Self-adaptive partial discharge signal de-noising based on ensemble empirical mode decomposition and automatic morphological thresholding," IEEE Transactions on Dielectrics and Electrical Insulation, vol. 21, no. 1, pp. 294-303, 2014.

[28] L. Shen, X. Zhou, L. Liu, and F. Yang, "Application of morphological wavelet de-noising in extracting gear fault feature," Evolutionary Intelligence, vol. 41, pp. 217-221, 2010.
[29] J. Cai and X. Wang, "Crack fault diagnosis of gear based on morphological wavelet de-noising," Jixie Qiangdu/Journal of Mechanical Strength, vol. 37, pp. 398-402, 2015. 